\title{
FERTILITY ANALYSIS AND RECOMMENDATIONS MANAGER
} (F.A.R.M.)

\author{
R.E. Karamanos, G.A. Kruger ${ }^{1}$ and J.L. Henry ${ }^{2}$ \\ 1Saskatchewan Soil Testing Laboratory and \\ ${ }^{2}$ Department of Soil Science, University of Saskatchewan \\ Saskatoon, Sask., S7N 0W0
}

\section{INTRODUCTION}

F.A.R.M. or Fertility Analysis and Recommendations Manager is a software package, which was introduced by the Saskatchewan Soil Testing Laboratory for the first time in the fall of 1991 to process farm soil testing. Implementation of the F.A.R.M. System also coincided with the implementation of the new system of nitrogen fertilizer recommendations developed by Henry $(1990 ; 1991)$. Phase I of the F.A.R.M. System regulates the whole farm soil testing process from receiving of a soil sample to printing of a soil test report. The system was developed in accordance with Quality Assurance procedures to maintain quality and integrity of soil sample processing through the laboratory as well as to allow for a smoother and more efficient flow of samples and information through the laboratory and better communication of results to the customer.

The System consists of five modules, namely, Data Entry, Analysis and Approval, Calculation Parameters, Sample Tracking and Calculation Options (Figure 1). The purpose of this presentation is to briefly describe each of these modules.

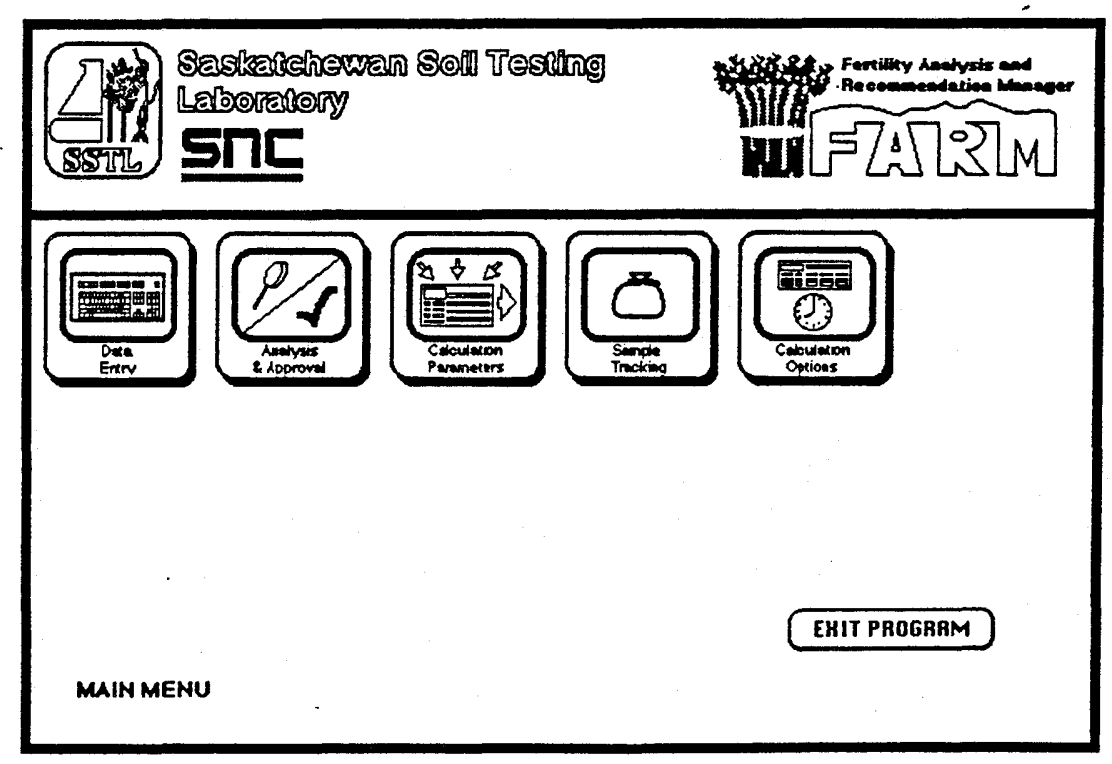

Figure 1. The five modules of the F.A.R.M. System. 


\section{DATA ENTRY}

The Data Entry module provides the user with the option of entering Information Sheets or results of analyses (Figure 2).

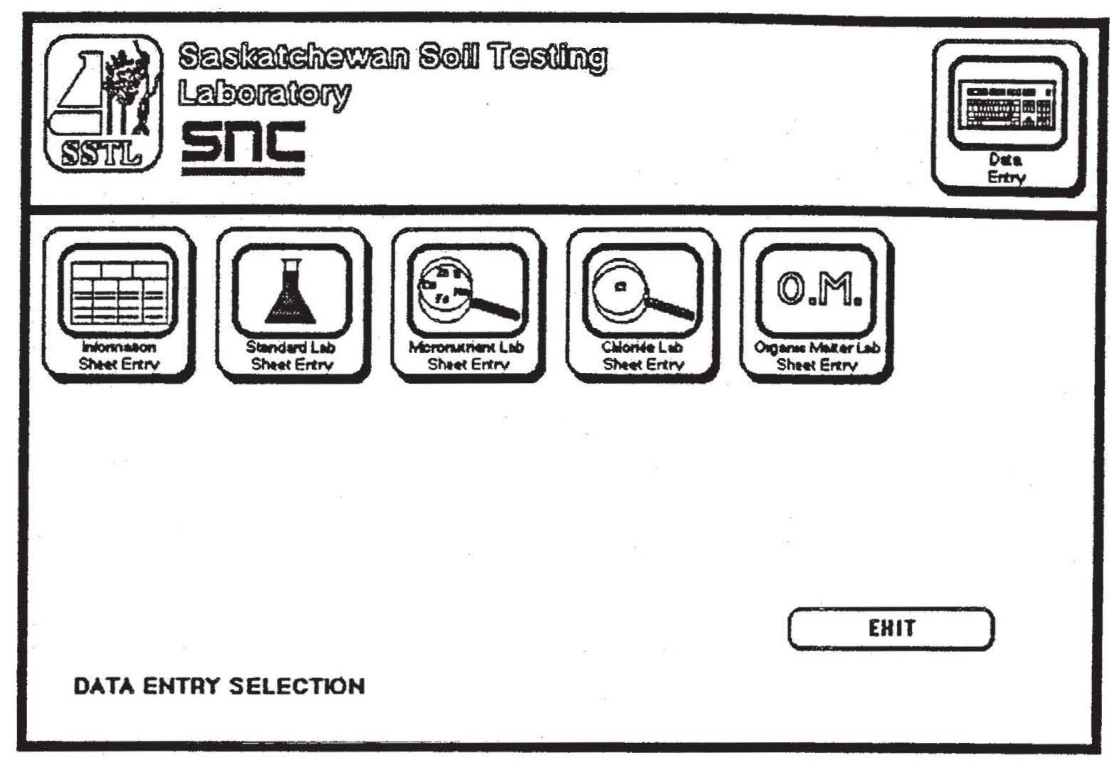

Figure 2. Data Entry selections in the F.A.R.M. System

The System allows for double entry only of all field information and analytical results. The field information or analytical results are entered by one data entry operator in one terminal and are re-entered by a second data entry operator in a second terminal. If discrepancies between the two entries are noted the computer requests that choice of the correct information is made and entered into the system. Only when information is double entered does the computer generate the corresponding receiving sheets and lab sheets for further processing of samples.

When entry of information sheet is completed, the computer shorts the fields on the basis of the number of depths samples from a field are submitted as (Figure 3 ) into single depth (0-6" or $0-12$ " or 0 -odd depth), double depth (0-6" and 6-12", 0-6" and 6-24" or 06" and 6-odd depth, or 0-12" and 12-24" or 12-odd depth) or triple depth (0-6", 6-12" and 12-24" or 12-odd depth) cases. A case contains a maximum of twenty four samples two or three of which, depending on the depth(s) submitted, are duplicates. Hence, a case of single depth fields will contain twenty-two fields (samples) and two duplicates, one of double depth fields eleven fields (twenty-two sampies) plus two duplicates, and one with triple depth fields seven fields (twenty-one samples) plus three duplicates. When partial cases are processed, the system selects one duplicate per eleven single depth fields or portion thereof, two duplicates of any number of double depth fields and three duplicates of any number of triple depth fields. Duplicates are selected randomly by the computer. Once shorting and selection of duplicates is completed, the computer assigns a number to each case and each sample and then prints a Receiving Sheet, a set of Labels and a Lab Sheet for each case. 


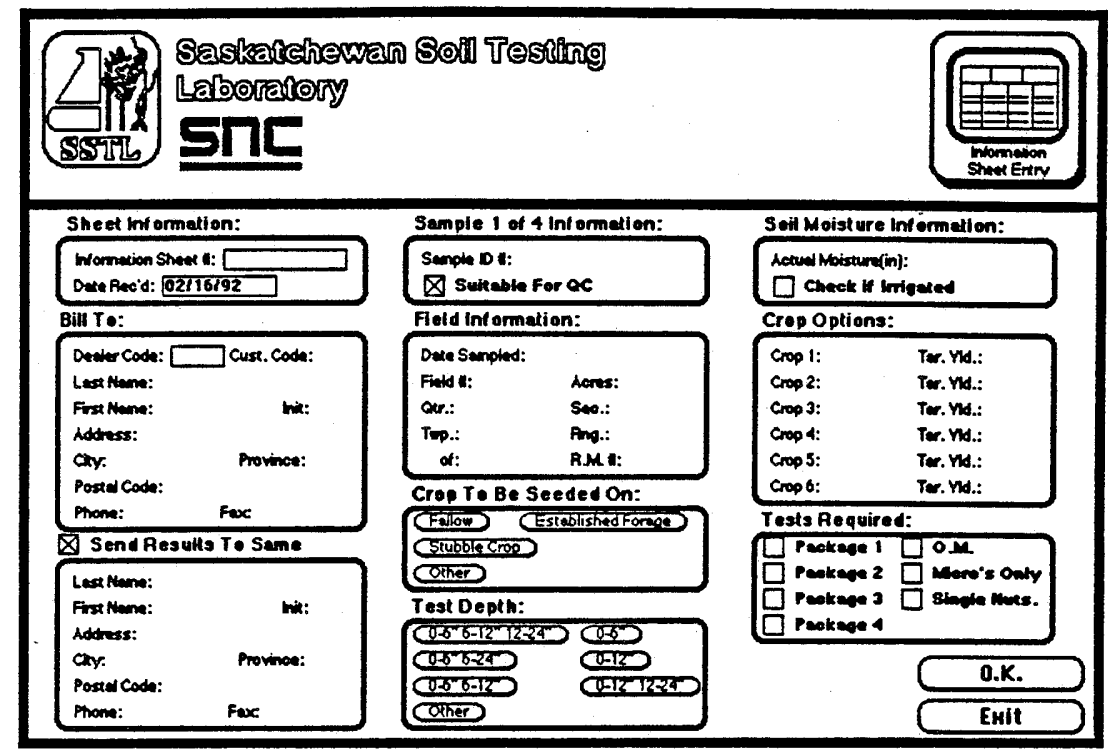

Figure 3. Information Sheet entry screen.

The Receiving Sheet contains the position of each of the samples in a case (1-24), which corresponds to that of a twenty-four position tray, the defsubmission and the Field I.D. (Figure 4). The set of labels contains the same information along with the date of receipt and when samples are to be discarded. The receiving department matches the labels with the Field I.D. on each of the soil sample bags. The case is then moved on to the Quality Control Assistant, who verifies the order of samples, splits the duplicates and replaces the Receiving Sheet with the Lab Sheet (Figure 5). From this step on, no one in the laboratory knows of the identity of the samples processed. The samples in two consecutive cases (forty eight) are supplemented by a blank for each extractant and a standard soil sample to complete a "set" of fifty samples processed through the laboratory.

Quality control is implemented by each analyst by ensuring that the results for the standard soil and those for the duplicates fall within control limits established by the Quality Control Manager. The process is facilitated through Q.C. charts for each nutrient or parameter analyzed. Audit and assessment of the quality control process is carried out daily by the Quality Control Manager.

The analytical results for the various tests performed on soil samples are entered by selecting the appropriate data entry sheet in the Data Entry Module (Figure 2). Again double entry of all analytical results is necessary.

The process described so far takes approximately two days to complete. 
SSTL F.A.R.M. Program

F.A.R.M. Program

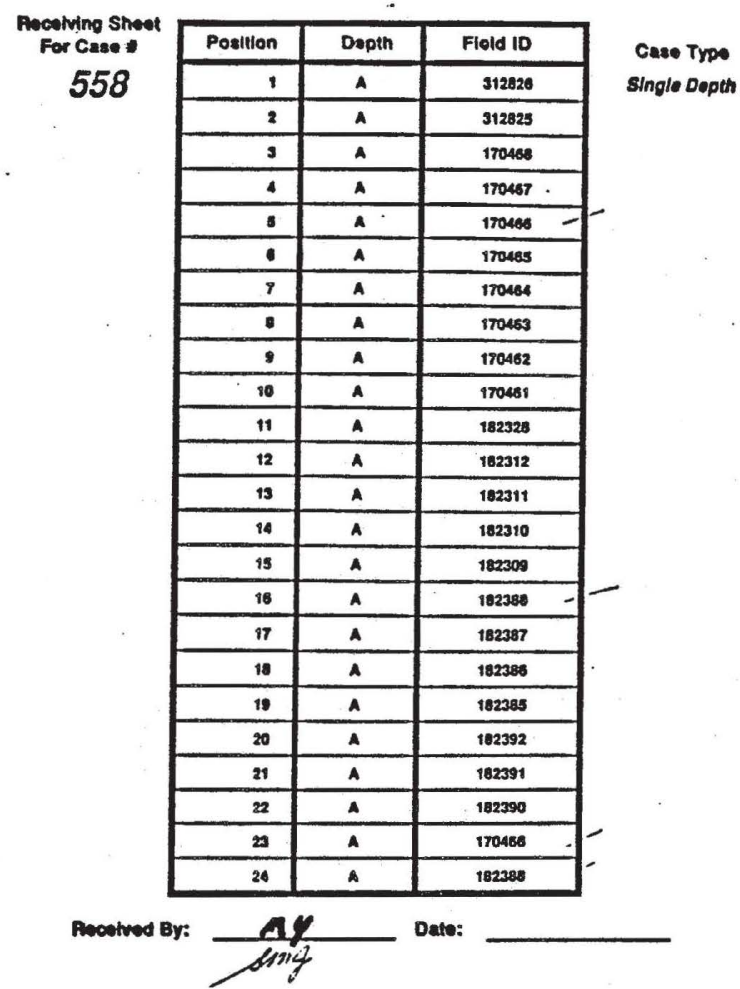

Figure 4. Example of a Receiving Sheet.

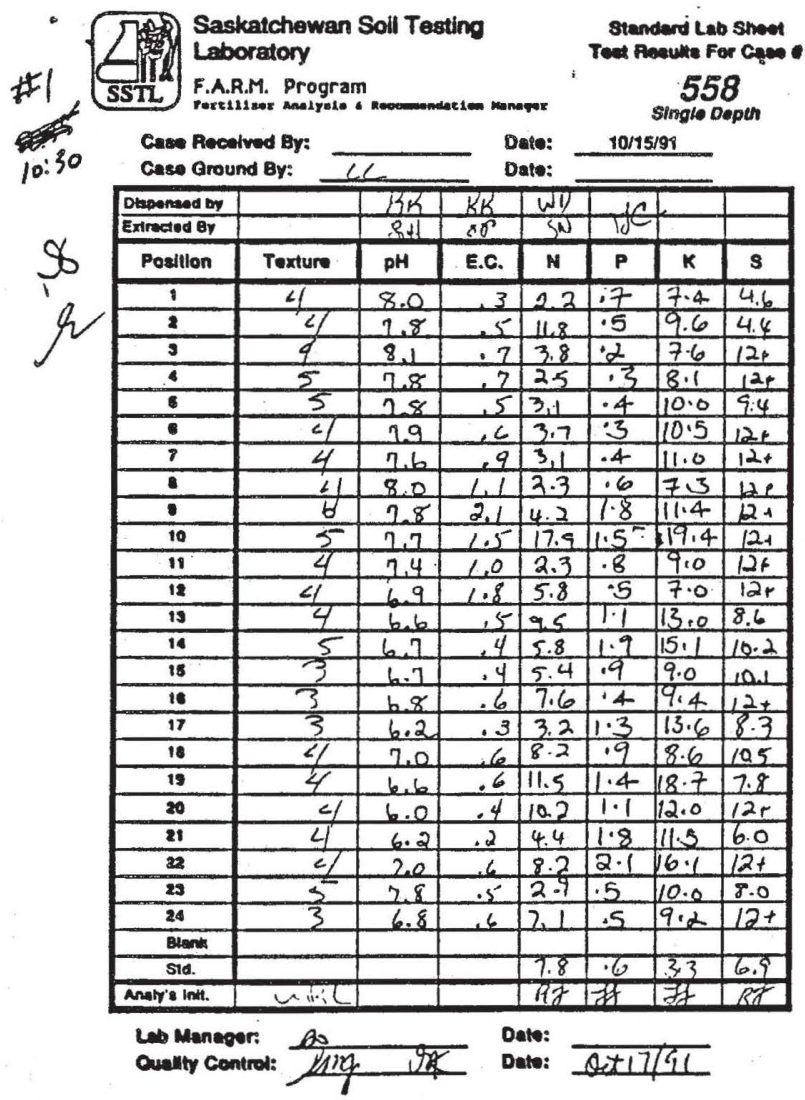

Figure 5. Example of a Standard Lab Sheet. 


\section{CALCULATION PARAMETERS}

This module processes all analytical data and prepares them for final processing and approval. The module contains all Standard Databases (crop types, previous crop practice, textures, soil climatic zones and dealer/agent codes) required to classify information, a set of Filters for screening of field information and analytical results, standard Comments and Recommendation Parameters (Figure 6).

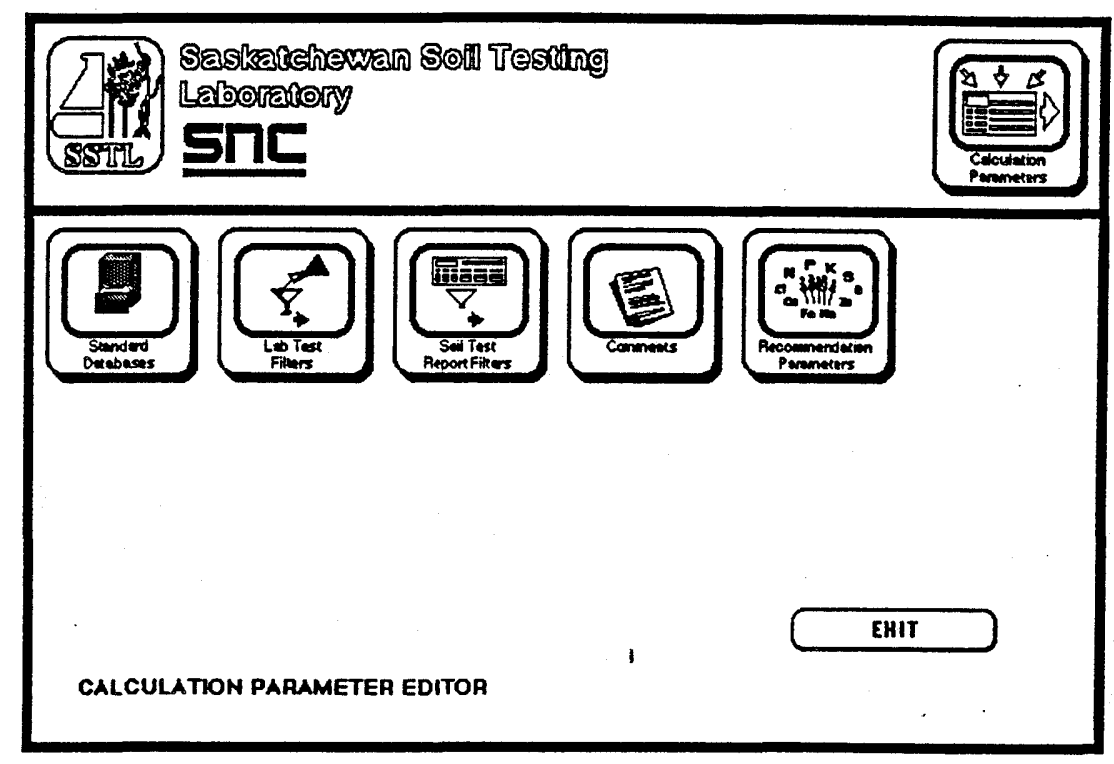

Figure 6. The Calculation Parameter module of the F.A.R.M. System.

The Standard Databases assign a set of recommendations for each crop type and for each nutrient. Each set of recommendations is either in the form of functions or as tables and is handled by the Recommendation Parameters. Recommendations also depend on whether a crop is to be seeded in a fallow of a stubble field or is an established forage.

A set of filters checks all analytical data to ensure conformity to the quality control limits for both the standard soil and the duplicates run for each case. In the event that an analyst overlooks a quality control failure, the system responds by pointing out the case where a failure occurred. If the failure has occurred in the standard soil, it rejects the case(s) and prints a new receiving sheet, lab sheet etc for re-processing the case(s) through the system. If the failure has occurred in the duplicate samples, it alerts the Quality Control Manager, who then has to manually ascertain the cause of the failure and decide whether the whole case has to be rejected.

Another set of filters allows for a "qualitative" agronomic check by establishing ranges beyond which results may be questionable. As an example, if a stubble soil with high levels of nitrogen is encountered, the system will alert the agronomist. An example of the agronomic filters is given in Figure 7. 


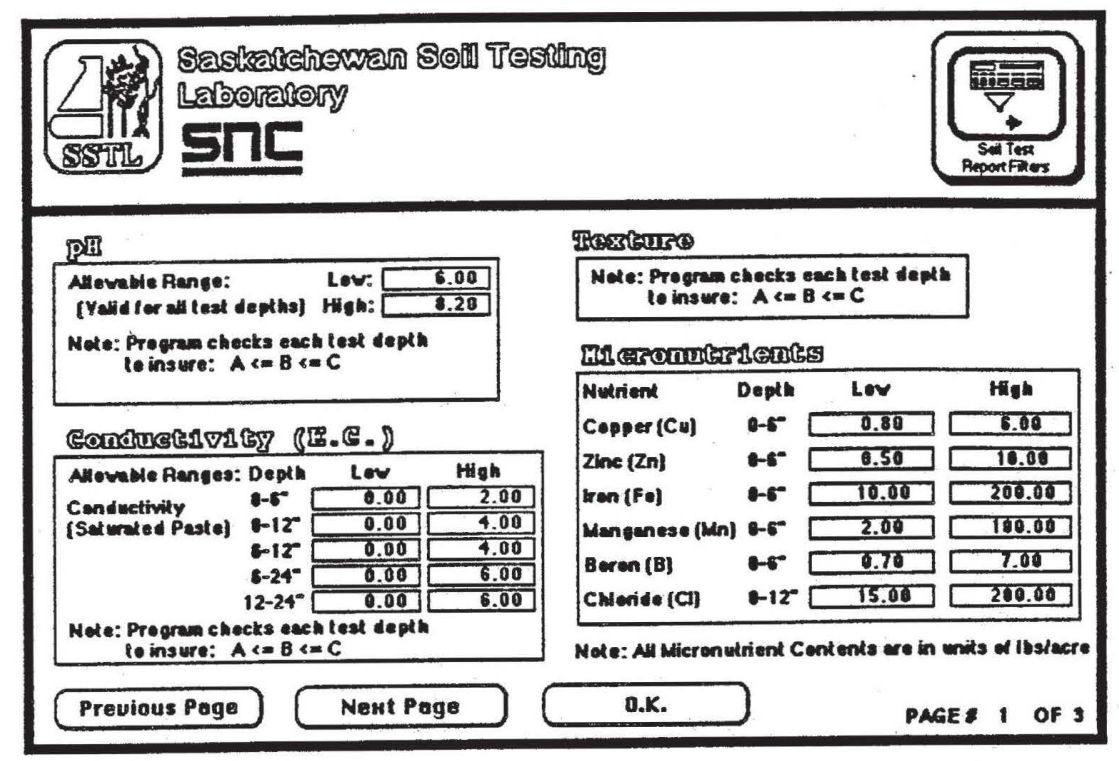

Figure 7. An example of "agronomic" filters contained in the F.A.R.M. System.

The System generates comments on the basis of a set of conditions identified through a number of parameters (Figure 8).

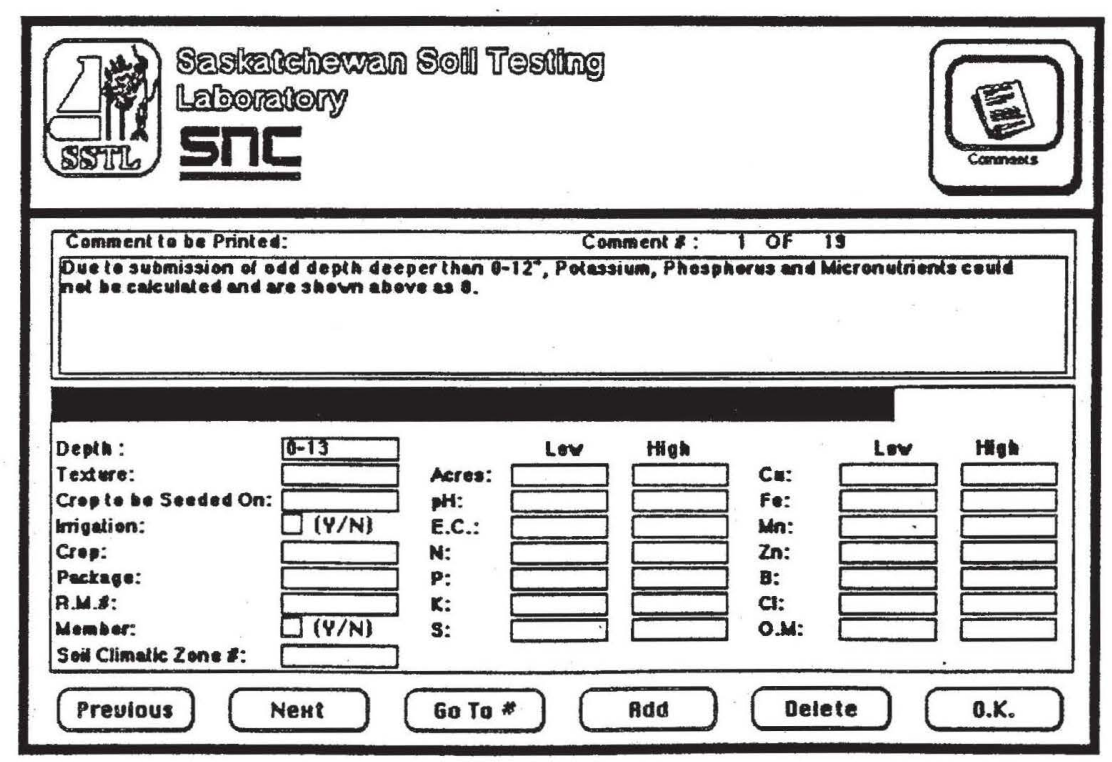

Figure 8. Example of entering conditional comments in the F.A.R.M. System. 
Once, analytical data have been converted to $\mathrm{lb} / \mathrm{ac}$, checked for quality control and passed through a set of preliminary agronomic checks, the recommendations for the crop(s) and set of conditions for each field are calculated. This step is performed in the Calculation Parameter module by the Recommendation Parameters (Figure 9).

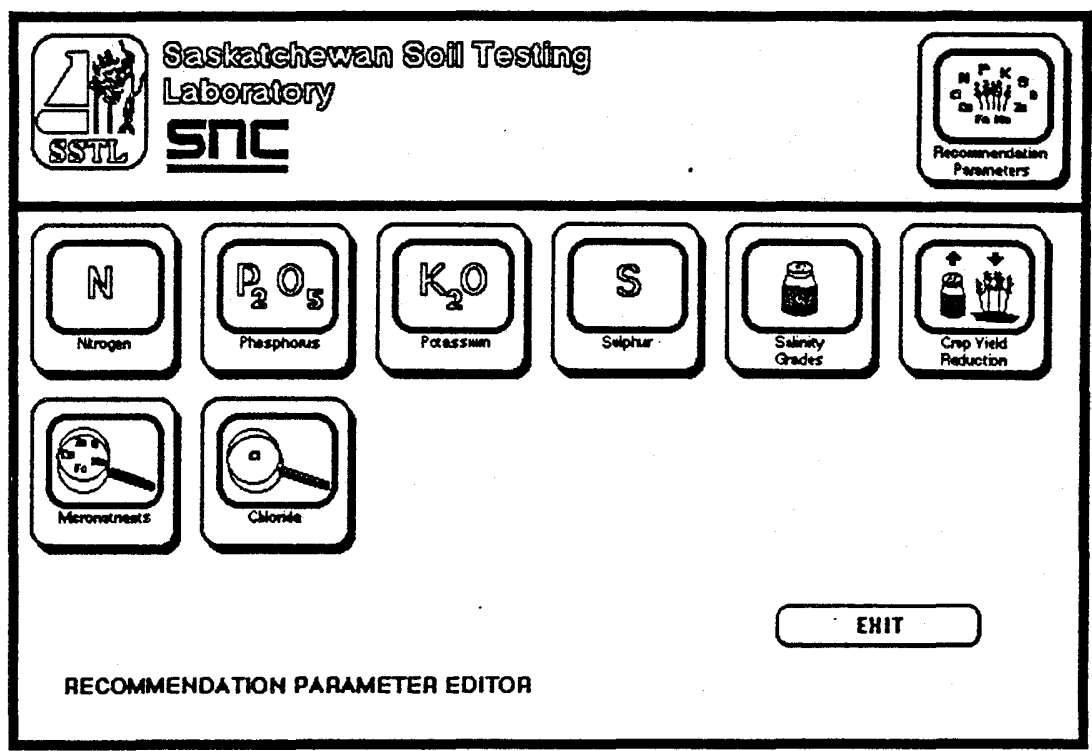

Figure 9. Recommendation Parameter Editor of the F.A.R.M. System.

Recommendations for all nutrients except Nitrogen are in a tabular form and are provided on the basis of the set of recommendations assigned to each crop in the Standard Databases. The recommendation tables include a range of soil test values for each nutrient, whether recommendations are for irrigated or dryland crops, a texture modifier for certain nutrients (e.g., phosphorus), and three sets of recommendations corresponding to Dry, Normal and Wet conditions (Figure 10).

- The database for Nitrogen recommendations is quite elaborate, since it contains all the parameters defined by Henry $(1990 ; 1991)$ as necessary in order to provide nitrogen recommendations based on water use efficiency functions (Figure 11). Thus, the Nitrogen Parameter Editor contains the Assumptions utilized by the system in calculating soil and fertilizer nitrogen use efficiency, nitrogen immobilization through straw additions and efficiency of mineralized nitrogen. Soil Climatic Zone (SCZ) Locations contain the soil climatic zone classification of every square township in the three prairie provinces. "Typical" values for soil moisture utilized for fall sampling are included in the SCZ Available Nitrogen/Moisture. Yield Equations contains the water use efficiency functions for those crops that pertinent information is available. If a yield equation has not yet been developed, recommendations for Dry, Normal and Wet conditions are included in tabular form under Yield Tables (Figure 11). Other parameters, such as nitrogen crop removal, mineralization as a function of precipitation for each Soil Climatic Zone etc are also included in the Nitrogen Parameter Editor. 


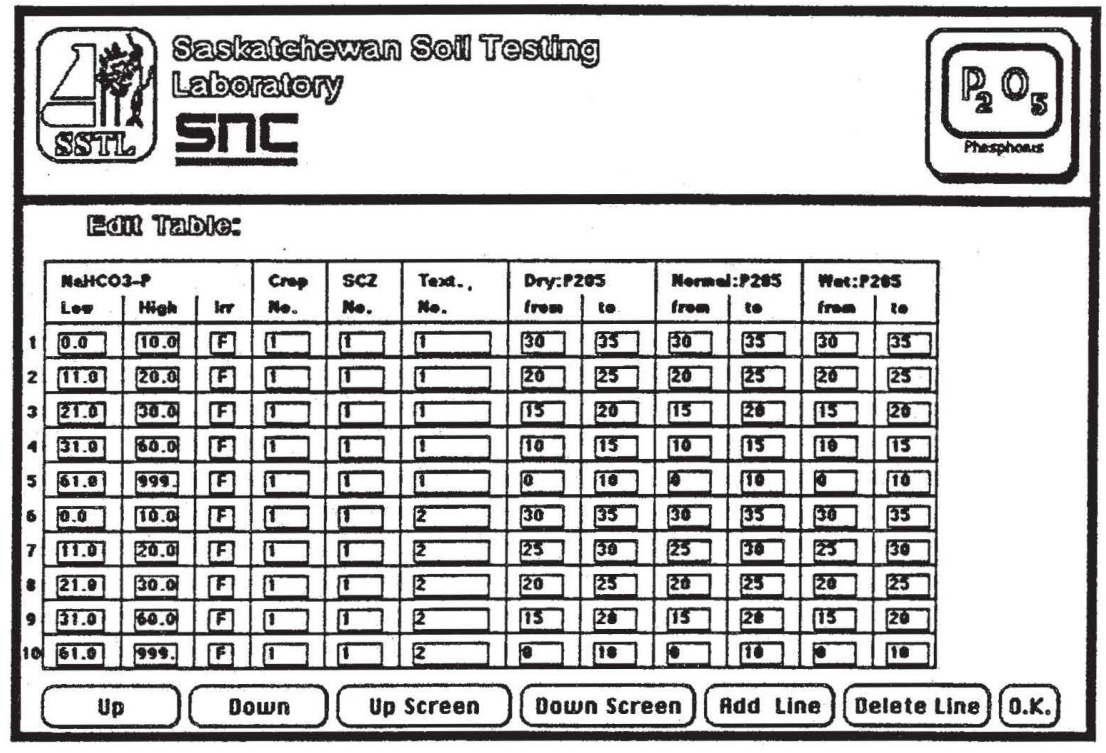

Figure 10. Example of a Recommendation Table for nutrients other than Nitrogen.

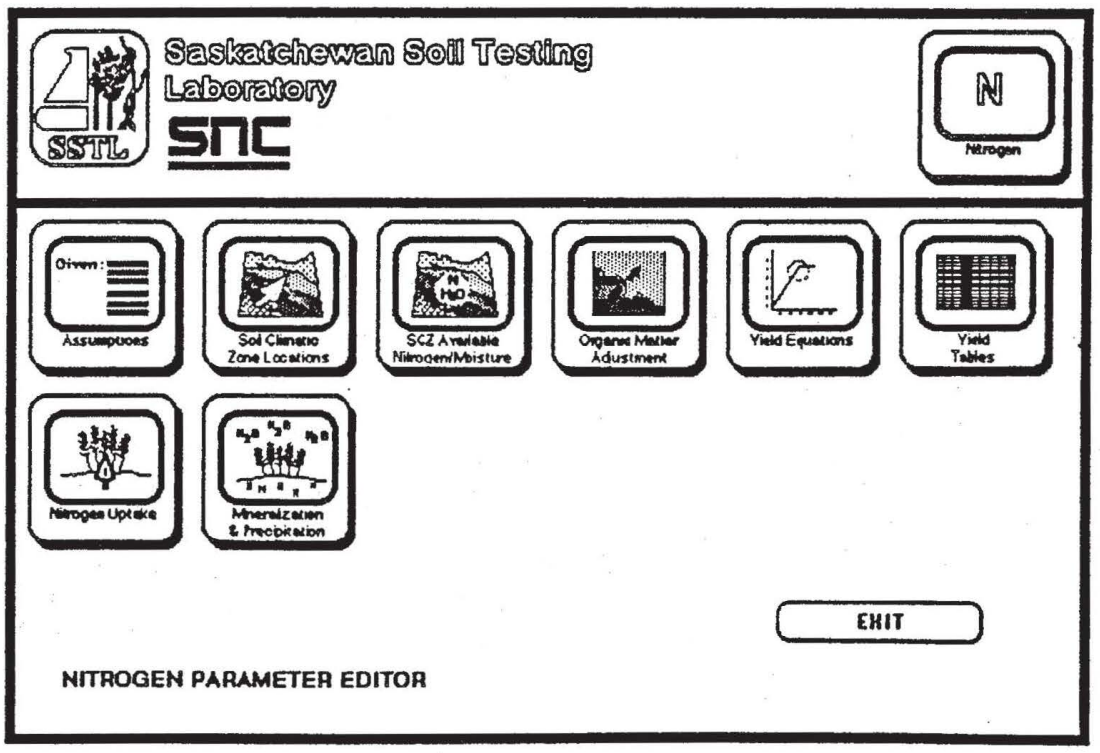

Figure 11. Nitrogen Parameter Editor of the F.A.R.M. System 


\section{SAMPLE TRACKING}

The progress of sample through the laboratory can be monitored at any time via the Sample Tracking module (Figure 12). A sample can be identified uniquely in the System by Field Number (i.e., the number on the information sheet). However, a sample can also be tracked down by the Dealer/Agent code, farmer name, phone or fax number or the number each information sheet is assigned, which also appears at the bottom left corner of a Soil Test Report. The System identifies the status of a sample in the laboratory as having been received but not analyzed, analyzed but not approved, approved but a report not printed, a summary report faxed but the final report not printed or a report faxed and mailed.

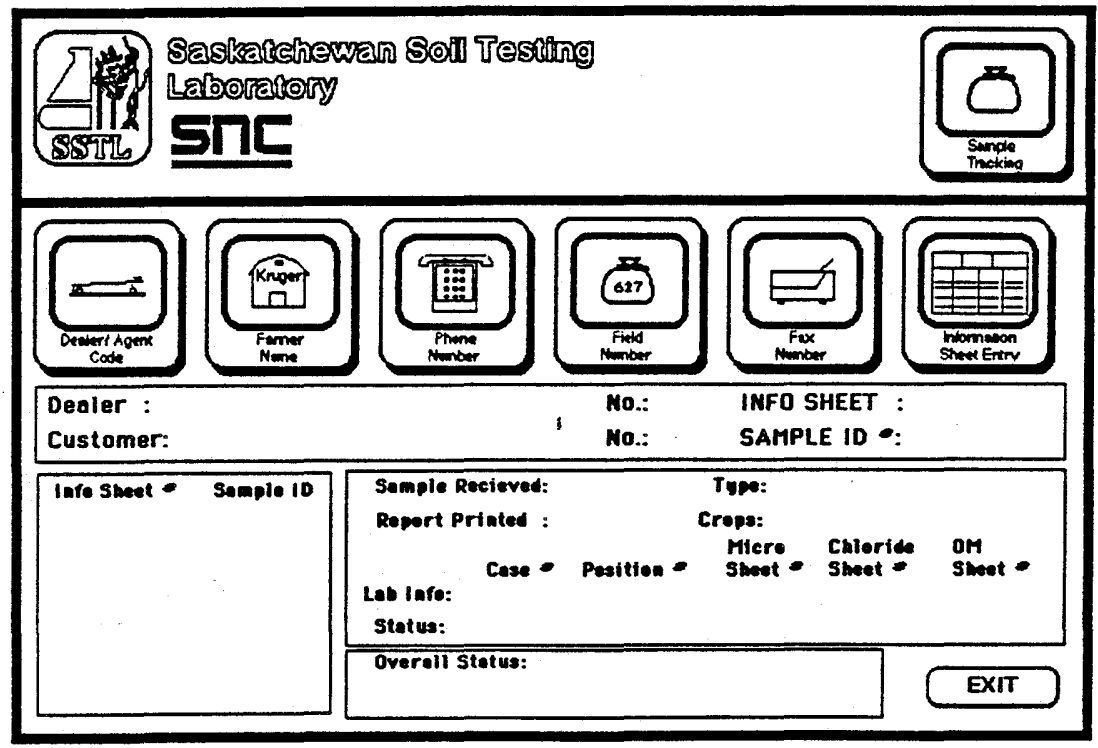

Figure 12. The sample tracking module of the F.A.R.M. System.

\section{ANALYSIS AND APPROVAL}

This module allows the Quality Control Manager and the Laboratory Agronomists for on screen scanning of all analytical result and recommendations, respectively. The status of each case, after it has passed through the Lab or Agronomic filters is displayed first on a Quality Control and then on an "agronomic" screen (Figure 13). Although results may have passed through the filters successfully, approval of each case by the Quality Control Manager and subsequently by an Agronomist is necessary before a case is released for final processing. If a case is rejected by either, lab sheets for all analyses associated with the soils of the case are generated and the case has to be re-received and re-processed. Passwords protect this module, which allow access only to qualified personnel (i.e., agronomic check can only be performed by a Professional Agrologist). 


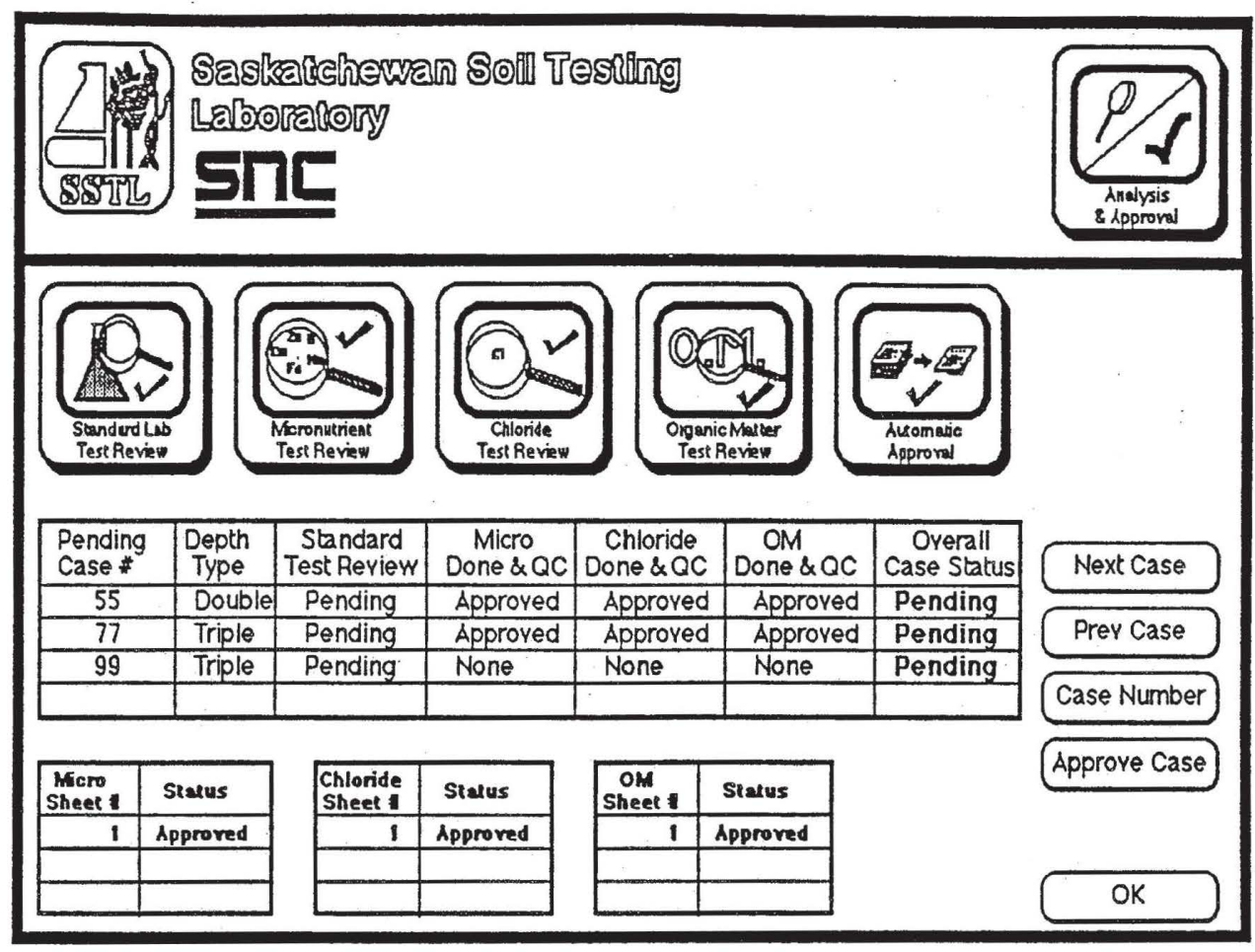

Figure 13. The Analysis and Approval module of the F.A.R.M. System.

I

\section{CALCULATION OPTIONS}

This module monitors calculations and reporting through the whole process. It provides options for printing Receiving and Lab Sheets, for running lab and agronomic filters and for faxing and printing soil test reports.

\section{REFERENCES}

Henry, J.L. 1990. Development of crop water production functions for wheat, barley and canola to revise nitrogen fertilizer recommendations in Saskatchewan. University of Saskatchewan, Saskatoon, Sask., Mimeo Report 16 pp.

Henry, J.L. 1991. Development of soil test based nitrogen fertilizer recommendations for wheat, barley, canola, flax oats. University of Saskatchewan, Saskatoon, Sask., Mimeo Report 10 pp. 